Urologe 2014 · 53:1134-1135

DOI 10.1007/s00120-014-3592-3

Online publiziert: 7. August 2014

(c) Springer-Verlag Berlin Heidelberg 2014

\author{
W. Bühmann \\ Facharzt für Urologie - Andrologie, Medizinische Tumortherapie - Qualitätsmanagement, Sylt OT Keitum
}

„Nu stellen wa uns ma janz doof und denken ma janz eenfach, wa...?" - unter unbewußter Berücksichtigung dieses Lieblingszitats des großen Berliner Entertainers Harald Juhnke (1929-2005) sind wohl die allermeisten unseres Standes zu Beginn ihrer ärztlichen Laufbahn angetreten, um ausschließlich dem individuellen, vertrauensvollen wie erfolgreichen diagnostischen und therapeutischen Dia$\log$ mit unseren Patienten zu dienen, ohne Frage unter Erwartung einer für Verantwortung und Arbeitsdichte angemessenen Vergütung. In die Berufspolitik und ärztliche Selbstverwaltung wurden viele Jahre lang eher die Kollegen entsandt, die im „Kernberuf“ weniger erfolgreich waren oder erkannt hatten, dass sie doch weniger Arzt als Funktionär und/oder Politiker sein wollten, um auch denen ein auskömmliches Leben zu ermöglichen.

Erst als auch die letzten ausschließlich patientenorientierten Kolleginnen Ende der 1980er Jahre begriffen hatten, dass die ",angemessene Vergütung" einer partiellen Enteignung gewichen war - Budgetierung seit 1989 bedeutete nicht nur faktische Halbierung des Fallwertes und Begrenzung auch der fallzahlabhängig möglichen Honorarsteigerung, sondern auch noch die Halbierung der Kaufkraft des erzielten Einkommens durch die Einführung des Euro 2002, deren Kompensation den Ärzten - anders als anderen „Dienstleistern“ durch Preisverdoppelung - verwehrt blieb, bedurfte es professionellerer berufspolitischer Strukturen, um dieser Entwicklung mit wirkungsvollerer Defensive zu begegnen als durch gemeinsame Arbeitsessen in stilvoller Atmosphäre.

Mein Großvater hat sich als chirurgischer Chefarzt (1919-1953) durch ein gu- tes Einkommen aus seiner Tätigkeit erlaubt, sozial schwache oder nicht ordentlich versicherte Patienten gratis zu operieren - die Dankbarkeit der Patienten war ihm sicher, auch manchmal durch z. B. eine "schwarz" geschenkte selbst gezogene Weihnachtsgans in Kriegszeiten. Mein Vater konnte als niedergelassener Internist (1953-1987) die schon damals nicht bezahlte Zuwendung zum Patienten durch die unbudgetierten $8 \mathrm{DM}$ für eine Gamma-GT quersubventionieren - beides heute nicht mehr möglich - warum nur?

Die Welt war in Ordnung, weil stillschweigend und selbstverständlich gesamtgesellschaftlich konsentiert war, dass Gesundheit Geld kosten darf und die, die sie unmittelbar für den Patienten gestalteten, dafür anständig bezahlt wurden. Dieser Konsens wurde seitens der Kostenträger und Politiker vor rund 25 Jahren grundlos aufgekündigt, obwohl es noch nie soviel Geld für Gesundheit gab wie heute; seitdem ist angesagt, durch jahrelange berufspolitische Kreativität zu versuchen, einen Teil der enteigneten Einkommen wieder zu akquirieren: „Wir nehmen Euch drei Viertel weg, und nun versucht mal, uns zu überzeugen, Euch für Mehrleistungen (sprich: Qualitätssicherung und -management, Selektivverträge, DMP-Programme, Praxisnetze, MVZ-Gründungen, DRG-Kodierungsbetrug, Personalabbau in Praxis und Klinik, Operationsprämien für Chefärzte, IVVerträge, Fehlermanagement, Arbeitszeitausweitung und Dokumentationsorgien etc.) mal 5, 10, 20 oder gar 35 EUR pro Patient und Vierteljahr zurückzugeben. Gleichzeitig "feiern“ wir Euch Ärzte als gierige Streiter, wenn es Euch gelingt, die von uns halbierten 10 EUR dann mit zwei- stelligen Zuwachsraten wieder auf 7 EUR zu steigern - so viele Prozente bekommt niemand zugestanden außer Euch..." - so lief die Diskussion zwischen David und Goliath in den letzten 25 Jahren ab.

Aber ein Gutes hat es: Die allermeisten dieser neu erfundenen Mehrleistungen dienen ja ausschließlich der Steigerung der Qualität für die Patienten (s. Liste oben) und keinesfalls der individuellen Lebensqualität des Arztes - von dieser Zielrichtung haben wir uns längst demütig verabschiedet.

Aus diesem Gesamtblick auf den Makrokosmos heraus begeben wir uns wieder in den urologischen Mikrokosmos des vorliegenden Heftes, das sich - gutem Brauch folgend unter der Schirmherrschaft der Präsidenten unserer beiden Verbände DGU und BDU - der aktuellen Berufspolitik widmet. Und so darf ich als (fast) unbefangener Beobachter konzedieren, dass tatsächlich inzwischen kompetente und motivierte Berufspolitiker auf dem mühsamen Weg der Schadensbegrenzung unterwegs sind. Davon mögen die folgenden neun Beiträge Zeugnis ablegen:

DGU-Präsident Jan Fichtner beleuchtet gemeinsam mit Andreas Schneider, Vorsitzender des BDU-Arbeitskreises Belegärzte „Die demographische Entwicklung in Deutschland: Herausforderung und Chance für die Urologie“, die in den nächsten Jahren erhebliche strukturelle Anstrengungen erfordern wird, um die bisher hervorragende Patientenversorgung in Klinik und Praxis zu sichern.

Dr. W. Bühmann ist Pressesprecher des Berufsverbandes der Deutschen Urologen e.V. und Mitglied der Schriftleitung „Der Urologe“. 
„Zertifizierungssysteme in der urologischen Versorgung" werden von BDUSchriftführer Richard Berges und BDUPräsident Axel Schroeder als Anreiz für Gedanken zur möglichen Zusammenführung im Sinne einheitlicher Maßstäbe zur Beurteilbarkeit einer Qualitätsklassifikation bewertet.

DGU-Generalsekretär Oliver Hakenberg und Axel Schroeder nehmen Stellung zu dem neu geplanten „Institut für Qualitätssicherung in der ambulanten und stationären Versorgung “, in dem - bisher ohne ärztliche Beteiligung - die Qualität in Diagnostik und Behandlung gemessen und bewertet werden soll.

Der Beitrag „Dokumentation in der ambulanten Uroonkologie - der Beitrag des IQUO e.V. zur Qualitätssicherung und Versorgungsforschung" von Matthias Schulze, 2. BDU-Vizepräsident und IQUO-Vorstandsmitglied, stellt eine wichtige Plattform für effektive Datenkommunikation und Wissenstransfer in den Fokus, die schon längst selbstverständlicher Bestandteil urologischer Verständigung hätte sein sollen.

Um die Versorgung der urologischen Patienten sowohl im Bereich der Behandlung des Prostatakarzinoms mit der Option "Active Surveillance“ als auch im Bereich „Pflege“ zu sichern und zu optimieren, hat der BDU bereits einen Vertrag abgeschlossen und steht in weiteren Verhandlungen. Den aktuellen Stand und die Bedeutung der Selektivverträge für die Versorgung dokumentieren Patricia Ex, Consultant bei WMP-Healthcare Berlin und Axel Schroeder in ihrem Beitrag dazu.

Die „Akademie der Deutschen Urologen“" entwickelt sich zur zentralen Institution für urologische Fortbildung in Deutschland - als gemeinsame Einrichtung von DGU und BDU. Die aktuelle Entwicklung 2014 erfahren Sie in dem Beitrag von Maurice S. Michel, Vorsitzender der Akademie, und den Vorstandsmitgliedern Oliver Hakenberg und Axel Schroeder.

BDU-Ehrenpräsident Klaus Schalkhäuser nimmt sich des Themas „Wahlleistungsrecht für Belegärzte“ an und differenziert die unterschiedlichen Möglichkeiten der Privatliquidationen für die versorgungswichtige Gruppe der über 400 urologischen Belegärzte.
„Delegation ja, Substitution nein!“ übertitelt Axel Schroeder seinen Beitrag zu den Überlegungen, inwieweit nichtärztliche Mitarbeiter(innen) Aufgaben übernehmen können/sollen, um die Fachärzte im Hinblick auf ihre individuelle Überlastung, aber auch zur Kompensation des bundesweiten Facharztmangels zu entlasten.

Zum Ende der Runde führt BDU-Pressesprecher Wolfgang Bühmann in die „never ending story“ der korrupten Ärzte in „Kooperation“ mit der Pharmaindustrie ein, der wir uns gemeinsam durch einen neu entwickelten Transparenzkodex stellen, um diesen würdelosen Attacken den Boden zu entziehen: „Neue Transparenz zwischen Ärzten und Industrie“.

Zusammenfassend hoffen alle Autoren, Ihnen einen informativen Querschnitt durch die derzeitigen gemeinsamen Aktivitäten unserer Fachgesellschaft mit dem Berufsverband zu geben, die sowohl fachintern die Zusammenarbeit fördern als auch in der Öffentlichkeit den Stellenwert unseres Faches deutlich machen sollen.

Viel Freude beim Lesen und Anreiz zur Diskussion wünscht Ihnen im Namen aller „Mittäter“

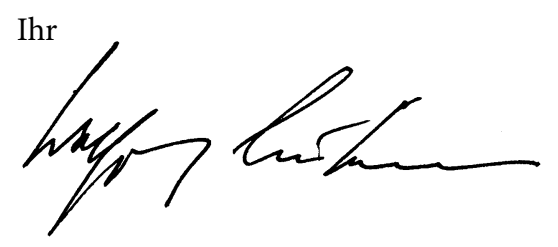

W. Bühmann

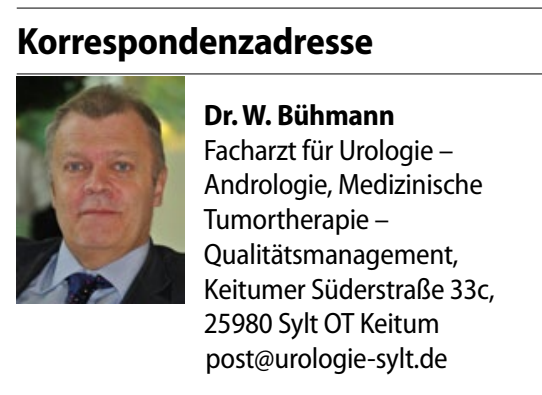

Interessenkonflikt. W. Bühmann gibt an, dass kein Interessenkonflikt besteht.

Dieser Beitrag beinhaltet keine Studien an Menschen oder Tieren.

\section{Ethik im Alltag - sinnvoll, hilfreich und machbar}

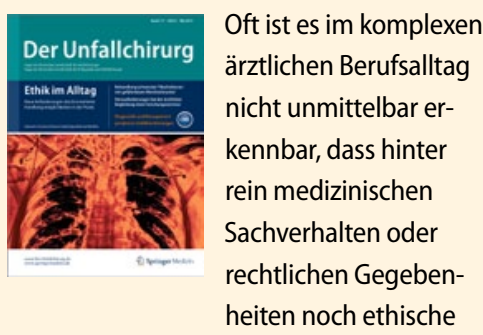

Fragen stehen könnten. Trotzdem, wenn es etwa um die Ausformulierung bestehender Aufklärungspflichten, um das Für und Wider einer konservativen oder operativen Behandlung oder um das Einstellen lebenserhaltender Sofortmaßnahmen im Schockraum geht, stößt man doch unvermeidlich auf ethische Kernfragen: Dürfen und sollen wir alles machen, was wir können? Nutzen oder schaden wir eher dem Patienten? Wie ist der Wille des Patienten einzuschätzen? Ausgabe 5/2014 von Der Unfallchirurg widmet sich folgenden Aspekten des komplexen Themas „Ethik im Alltag":
- Ethische Entscheidungen in der Chirurgie
- Änderung des Therapieziels bei kritisch Kranken
- Kostenbewusste ärztliche Entscheidungen
- Unterschiede in der Häufigkeit von Gesundheitsleistungen - ist alles unethisch?

Bestellen Sie diese Ausgabe zum Preis von 37,- EUR zzgl. Versandkosten bei Springer Customer Service Center Kundenservice Zeitschriften

Haberstr. 7

69126 Heidelberg

Tel.: +49 6221-345-4303

Fax: +49 6221-345-4229

E-Mail: leserservice@springer.com

Suchen Sie noch mehr zum Thema? Mit e.Med, dem Online-Paket von Springer Medizin, können Sie schnell und komfortabel in über 500 medizinischen Fachzeitschriften recherchieren.

Weitere Infos unter springermedizin.de/ eMed. 